\title{
Early versus delayed postoperative oral hydration in children following general anesthesia: a prospective randomized trial
}

\author{
Xiaorong Yin ${ }^{1}$, Xiaoqi Zeng ${ }^{1}$, Ting Wang ${ }^{1}$, Binbin Dong ${ }^{1}$, Menghang $\mathrm{Wu}^{1}$, Anna Jia ${ }^{1}$ and Ling Ye ${ }^{2^{*}}$
}

\begin{abstract}
Background: Oral hydration has typically not been administered for between 4 and $6 \mathrm{~h}$ postoperative for children's safety in China. But children are more likely to suffer from apnea, crying and agitation, wound bleeding, and other complications during the post-anesthesia recovery period because of thirsty and fear. This Prospective, randomized study sought to assess the compare the early and late oral hydration ( $\mathrm{EOH}$ and $\mathrm{DOH}$, respectively) in children following general anesthesia, with the goal of assessing relative safety and tolerability and thereby improving patient comfort.

Methods: A total of 2000 children corresponding to the American Society of Anesthesiology (ASA) I-III were randomized into an $\mathrm{EOH}$ group $(n=1000)$ and a DOH group $(n=1000)$. For the former group, children were administered a small amount of drinking water following recovery of the swallowing reflex, and children's vital signs were monitored for $20 \mathrm{~min}$ in a postanesthesia care unit (PACU). DOH group patients received water at $4 \mathrm{~h}$ following general anesthesia). All patients underwent monitoring to assess their thirst, satisfaction, oropharyngeal discomfort, nausea, and vomiting.
\end{abstract}

Results: Complete data were collected from a total of 1770 patients $(E O H=832, \mathrm{DOH}=938)$ and was compared via chi-squared and t-tests as appropriate. There was no hypoxemia in either group, nor did the incidence of nausea and vomiting differ between the two groups $(P>0.05)$. The thirst score of the $\mathrm{EOH}$ group was significantly decreased relative to the $\mathrm{DOH}$ group in the children over 5 years old after drinking for 10 to $20 \mathrm{~min}(P<0.05)$.

Conclusions: For children undergoing general anesthesia, a small amount of drinking water in the early stages of recovery will not increase the incidence of nausea, vomiting, or hypoxemia, but will decrease thirst and improve satisfaction. It is important, however, that medical staff carefully monitor the swallowing reflex and vital signs of all children.

Trial registration: This study was registered on the Chinese Clinical Trial Registry (ChiCTR-IOR-16008197) (http:// www.chictr.org.cn/index.aspx. On April 2, 2016 the first patients was enrolled and on March 31, 2016 the trial was registered).

Keywords: General anesthesia, Oral intake, Postoperative nausea, Vomitting

\footnotetext{
*Correspondence: zerodq_hx@163.com

${ }^{2}$ Department of Pain Management, West China Hospital, Sichuan University,

Chengdu, Sichuan Province 610041, People's Republic of China

Full list of author information is available at the end of the article
}

(c) The Author(s). 2020 Open Access This article is licensed under a Creative Commons Attribution 4.0 International License, which permits use, sharing, adaptation, distribution and reproduction in any medium or format, as long as you give appropriate credit to the original author(s) and the source, provide a link to the Creative Commons licence, and indicate if changes were made. The images or other third party material in this article are included in the article's Creative Commons licence, unless indicated otherwise in a credit line to the material. If material is not included in the article's Creative Commons licence and your intended use is not permitted by statutory regulation or exceeds the permitted use, you will need to obtain permission directly from the copyright holder. To view a copy of this licence, visit http://creativecommons.org/licenses/by/4.0/ The Creative Commons Public Domain Dedication waiver (http://creativecommons.org/publicdomain/zero/1.0/) applies to the data made available in this article, unless otherwise stated in a credit line to the data. 


\section{Background}

Compared with adult patients, children are more likely to suffer from apnea, crying and agitation, wound bleeding, and other complications during the post-anesthesia recovery period because of their own physiological and psychological characteristics [1-3]. Crying and agitation during the recovery period may cause airway spasms, severe hypoxemia leading to respiratory depression or apnea, postoperative bleeding, accidental falls, and other adverse events. It therefore represents a safety concern during anesthesia recovery period, and remains a difficult problem for nursing care [4]. Through several years of clinical observation, we found that hunger and thirst were the primary causes of agitation in children.

In order to ensure postoperative safety and prevent coughing, vomiting, and aspiration caused by drinking water following general anesthesia for nongastrointestinal operations, postoperative oral hydration has typically not been administered for between 4 and $6 \mathrm{~h}$ in China [2, 3]. However, many researchers have found more rapid administration of oral hydration to be beneficial and safe [5-10]. The optimal timing for such administration of fluids, however, has remained unclear in China. For example, certain researchers have suggested that in children undergoing relatively minor operations water could safely be provided $1 \mathrm{~h}$ after anesthesia [8,9]. This study sought to compare early and delayed oral hydration (EOH and $\mathrm{DOH}$, respectively) after general anesthesia in order to assess the relative safety of these approaches, and to gauge their ability to decrease thirst and improve satisfaction and high-quality nursing service. Thirst, oropharyngeal discomfort, satisfaction, and incidence of nausea/vomiting, as well as the time from PACU arrival to first oral fluid intake, served as the primary outcome measurements for this study. Data was additionally collected regarding nausea and vomiting after returning to the ward, the incidence of postoperative complications, and the PACU observation time and hospitalization time.

\section{Materials and methods \\ Sample}

This prospective, randomized, controlled trial received approval from the Clinical trial and Biomedical Ethics Committee, West China Hospital, Sichuan University, Chengdu, China. This study was registered on the Chinese Clinical Trial Registry (ChiCTR-IOR-16008197) (http://www.chictr.org.cn/index.aspx. On April 2, 2016 the first patients was enrolled and on March 31, 2016 the trial was registered). From April 2016 - March 2018, a computer-generated list of random numbers served to mediate prospective randomization of a total of 2000 children corresponding to the American Society of Anesthesiology (ASA) I-III status who were in a postanesthesia care unit (PACU) after general anesthesia. Exclusion criteria included: preoperative colds, respiratory infections, facial, oropharyngeal, thoracic, gastrointestinal, neuro-, or laryngeal surgery, altered mental state, or dysphagia.

On the basis of pilot study, a sample size of 628 allowed a $20 \%$ difference between the two groups, with an $\alpha$ of 0.05 (two-tailed) and a $\beta$ of 0.20 , power of 0.8 . to account for attrition, a sample size of 785 was selected for each group.

\section{Study design}

For all study participants, a complete medical history was taken, and patients and their parents were then informed of the study protocols (including the hydration regimens, follow-up plans, and thirst/satisfaction scoring systems). We additionally informed both groups of possible complications associated with the $\mathrm{EOH}$ treatment, such as nausea or vomiting. We then obtained written informed consent from the parents of all participating children. After surgical treatment was complete, patients were transferred to the PACU where they were monitored via pulse oximetry, electrocardiography, and noninvasive blood pressure monitoring. Patients were also provided with fluid intravenously. Thirst was rated verbally using a scale of $0-100$, with the former representing no thirst and the latter representing the most significant possible thirst. Oropharyngeal discomfort similarly used a $0-$ 100 scale, with 0 representing comfort and 100 representing the most worst discomfort ever suffered. One day postoperatively, patient satisfaction regarding their assigned treatment regimen was also assessed on a 0-100 scale from not satisfied to most satisfied.

A total of 2000 patients were randomized into the $\mathrm{EOH}$ and DOH groups $(n=1000 /$ group). For the $\mathrm{EOH}$ group, medical professionals in the PACU monitored patient recovery until patients exhibited a normal mental status, degree $\mathrm{V}$ muscle recovery, stable vitals, and a normal swallowing reflex, at which time children were asked to consume water $(<5 \mathrm{ml} /$ $\mathrm{kg}, 5 \% \mathrm{GS})$. The water was administered by a trained doctor or nurse by first aspirating the appropriate amount of water in an empty needle according to the child's weight, tilting the child's head to one side, and then slowly administering the water in the corner of the mouth. Patients in the $\mathrm{DOH}$ group were given oral water $4 \mathrm{~h}$ following anesthesia according to standard protocols. Immediately following recovery from anesthetization, patients were assessed for thirst and discomfort. 


\section{Measures}

Primary outcome and secondary outcome were recorded. Children over 5 years of age used self-reporting scales for thirst and hunger, with both scales extending from 0 to 100 (0: not hungry/thirsty; 100: very hungry/ thirsty).

\section{Statistical analysis}

SPSS v18.0 (SPSS Inc., Chicago, IL) was used for all analyses. Data are given as means \pm SD. Continuous values were compared via Student's t-tests and ANOVAs, while categorical variables were compared via chi-squared tests with Fisher's test. $P<0.05$ was the significance threshold.

\section{Results}

A total of 2000 children were enrolled in this study, with complete data being available for 1770 of these study participants. Of the remaining patients, 88 were excluded due to a failure to drink following anesthesia, while 142 were excluded due to their unavailability for follow-up (Fig. 1). Table 1 summarizes the demographics of the patients included in the final study dataset, with no significant differences in age, gender, weight, surgical operation, or ASA status between groups.

Rates of nausea and vomiting were comparable between the $\mathrm{DOH}$ and $\mathrm{EOH}$ groups $(P>0.05)$ (Table 2$)$. A similar finding was observed with respect to oropharyngeal discomfort scales $(p<0.0001)$. EOH group patients exhibited higher postoperative satisfaction scores $(P<0.001)$ (Table 3$)$. With respect to the thirst and hunger scores of children over 5 years of age, compared with the DOH group, the scores of thirst and hunger in the $\mathrm{EOH}$ group at 10 and $20 \mathrm{~min}$ after general anesthesia were significantly lower $(P<0.05)$. After the patients were sent back to the ward, the thirst and hunger in the $\mathrm{EOH}$ group were significantly lower than those in the DOH group $(P<0.05)$ (Table 3$)$.

\section{Discussion}

The results of this randomized study indicate that $\mathrm{EOH}$ is safe in children immediately after general anesthesia associated with non-gastrointestinal surgery, significantly reducing thirst and discomfort while increasing satisfaction.

Postoperative hunger and thirst in children primarily occur due to prolonged preoperative fasting. In order to prevent reflux and aspiration during the operation, fasting for $2 \mathrm{~h}$ before anesthesia is necessary [9].

In this study, after recovery from general anesthesia, a small amount of water was given to children, and it did not increase the incidence of postoperative nausea or vomiting. This $\mathrm{EOH}$ treatment effectively lowered the thirst and hunger of children in the PACU, improving their comfort. Xiaoping Chen et al. found that the postoperative fasting time for patients undergoing non-

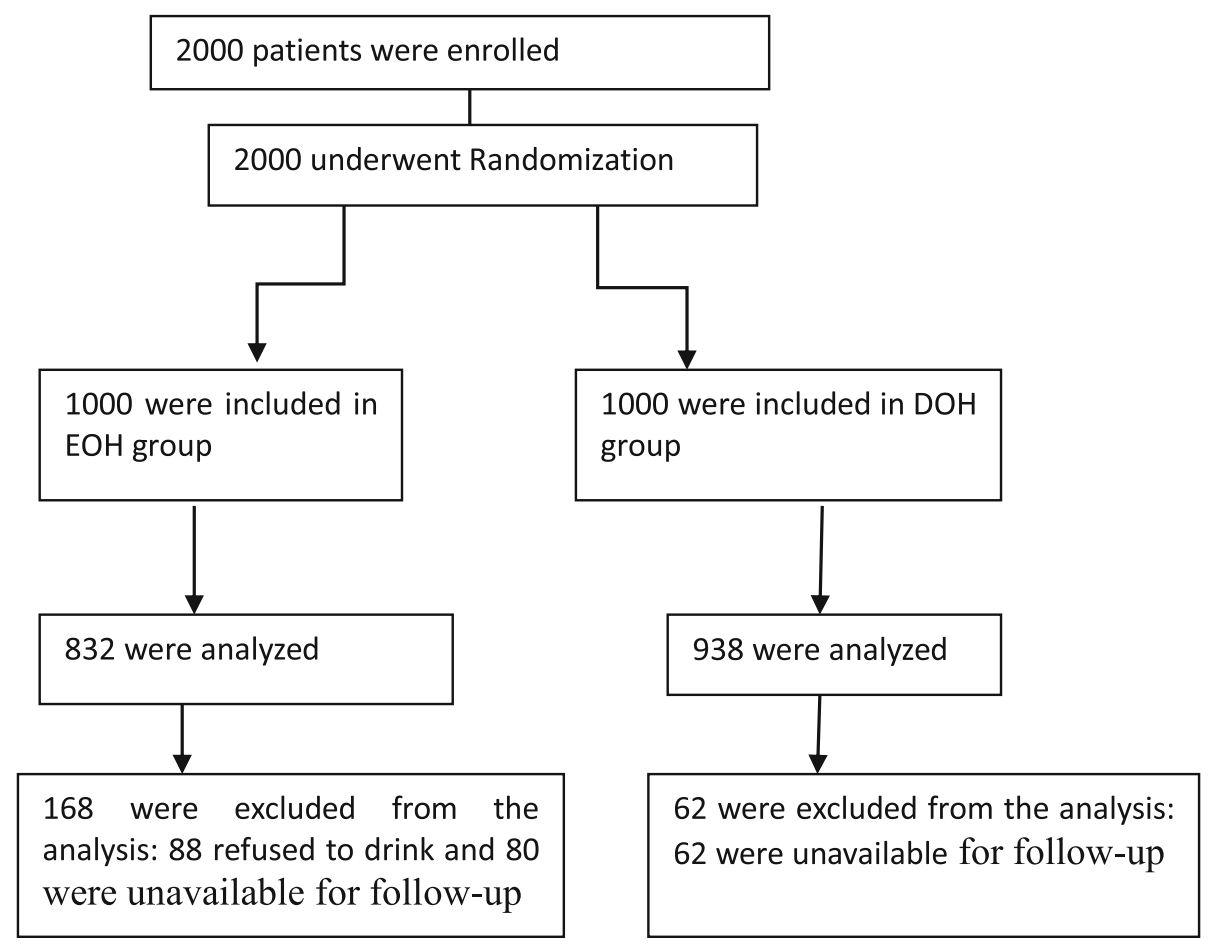

Fig. 1 Randomization, treatment, and inclusion in Analysis. EOH group: early oral hydration group; DOH group: delayed oral hydration group 
Table 1 Characteristics of the patient population and the surgical procedures performed

\begin{tabular}{|c|c|c|c|}
\hline Characteristic and surgical procedure & $\begin{array}{l}\text { Early oral hydration group } \\
(n=832)\end{array}$ & $\begin{array}{l}\text { Delayed oral hydration group } 4 \mathrm{~h} \text { after operation group } \\
(n=938)\end{array}$ & $P$ \\
\hline Age (y) & $5.59 \pm 3.25$ & $5.55 \pm 3.21$ & 0.79 \\
\hline Sex (\%) & & & 0.44 \\
\hline Male & $483(58.1)$ & $562(59.9)$ & \\
\hline Female & $349(41.9)$ & $376(40.1)$ & \\
\hline Weight (Kg) & $21.51 \pm 9.87$ & $21.59 \pm 10.20$ & 0.87 \\
\hline Anesthesia time (min) & $80.99 \pm 51.45$ & $78.53 \pm 48.13$ & 0.30 \\
\hline ASA (\%) & & & 0.88 \\
\hline । & $179(21.5)$ & $194(20.7)$ & \\
\hline$\|$ & $645(77.5)$ & $736(78.5)$ & \\
\hline III & $8(1.0)$ & $8(0.9)$ & \\
\hline Type of peration & & & 0.55 \\
\hline Polydactyly surgery & $13(1.6)$ & $26(2.8)$ & \\
\hline Burn and plastic surgery & $149(17.9)$ & $145(15.5)$ & \\
\hline orthopedic surgery & $239(28.7)$ & $256(27.3)$ & \\
\hline Hemangioma surgery & $22(2.6)$ & $29(3.1)$ & \\
\hline Ear surgery & $43(5.2)$ & $49(5.2)$ & \\
\hline Ophthalmologic surgery & $271(32.6)$ & $332(35.4)$ & \\
\hline Clubfeet surgery & $6(0.7)$ & $11(1.2)$ & \\
\hline Ambulatory Stitches remove & $12(1.4)$ & $11(1.2)$ & \\
\hline Hypospadias surgery & $75(9.0)$ & $76(8.1)$ & \\
\hline Double J stent remove & $2(0.2)$ & $3(0.3)$ & 0.75 \\
\hline
\end{tabular}

ASA American Society of Anesthesiologists

Table 2 Drugs used during general anesthesia

\begin{tabular}{llll}
\hline Drugs & $\begin{array}{l}\text { Early oral hydration group } \\
(n=832)\end{array}$ & $\begin{array}{l}\text { Delayed oral hydration group } \\
(n=938)\end{array}$ & $823(87.7)$ \\
\hline Propofol (\%) & $742(89.2)$ & $796(84.9)$ & 0.37 \\
Fentanyl (\%) & $707(85.0)$ & $790(84.2)$ & 1.00 \\
Midazolam (\%) & $717(86.2)$ & $938(100)$ & 0.26 \\
Sevoflurane (\%) & $832(100)$ & $77(8.2)$ & 1.00 \\
Sulfentanyl (\%) & $64(7.7)$ & $600(64.0)$ & 0.73 \\
Remifentanil (\%) & $163(19.6)$ & $6(0.6)$ & 0.59 \\
Cisatracurium Besilate (\%) & $534(64.2)$ & $5(0.5)$ & 0.96 \\
Succinylcholine chloride (\%) & $5(0.6)$ & $3(0.3)$ & 1.00 \\
Vecuronium bromide (\%) & $1(0.1)$ & $168(17.9)$ & 0.22 \\
ketamine (\%) & $1(0.1)$ & $748(79.7)$ & 0.63 \\
Ropivacaine (\%) & $122(14.7)$ & $3(0.3)$ & 0.07 \\
Ondansetron Hydrochloride (\%) & $656(78.8)$ & $330(35.2)$ & 0.68 \\
Tropisetron Hydrochloride (\%) & $4(0.5)$ & & 0.71 \\
Neostigmine (\%) & $259(31.1)$ & 0.08 \\
\hline
\end{tabular}


Table 3 Incidence of the postoperative nausea and vomiting in PACU and ward

\begin{tabular}{llll}
\hline & $\begin{array}{l}\text { Early oral hydration group } \\
(n=832)\end{array}$ & $\begin{array}{l}\text { Delayed oral hydration group } \\
(n=938)\end{array}$ & $\begin{array}{c}\text { P } \\
\text { PACU nausea after } 20 \text { min (\%) }\end{array}$ \\
PACU: vomiting after 20 min (\%) & $1(0.1)$ & $2(0.5)$ & 0.22 \\
Ward: nausea after 4h (\%) & $1(0.1)$ & $11(1.2)$ & 1.00 \\
Ward: vomiting after $4 \mathrm{~h}(\%)$ & $6(0.7)$ & $7(0.7)$ & 0.47 \\
\hline
\end{tabular}

abdominal surgery should be determined according to operation size, anesthesia method, and patient's reactions following general anesthesia, with patients eating following recovery and elimination of nausea/vomiting symptoms [10]. Another study by Ding Chuanzhong et al. [11], included a control group given a gradually increasing fluid diet $6 \mathrm{~h}$ after operation, as well as a control group in which children without nausea and vomiting were evaluated and given warm water after they had recovered from general anesthesia. These children were first given $15-30 \mathrm{ml}$ water, and, if they did not experience discomfort, some milk 15-30 min later, followed by glutinous rice porridge or noodles. In this study, the incidence of postoperative hyperpyrexia, thirst, hunger, crying, agitation, and family anxiety in the experimental group was significantly lower than that in the control group. We previously found it to be feasible for children to drink a small amount of GS after general anesthesia [12]. This work suggested that it was feasible and safe to drink a small amount of water after the recovery from general anesthesia once the cough and swallowing reflex had recovered.

Some researchers [13] have found that complete recovery of the swallowing reflex occurs within $24 \mathrm{~min}$ after anesthetization with propofol. Kerz et al. [14] concluded that it was safe to eat via the oral route at early time points after anesthetization when propofol is the only anesthetic used. Children have stomachs that empty rapidly, and preoperative fasting leads to hunger, crying, and significant swallowing of air, thereby leading to increased pressure in the stomach, abdominal distension, prolonged recovery of gastrointestinal peristalsis, and delayed recovery [15].

It is important that water intake in children be carefully monitored following general anesthesia. Children must be completely awake, with a recovered cough and swallowing reflex, as well as muscle strength that has recovered to grade V. When drinking water, children's heads should be tilted to one side, and children should be monitored for cough, nausea, and hypoxemia. There were no instances of hypoxemia during this study. Doctors or nurses must carefully conduct this water administration, monitoring patient vital signs at all times. A small amount of water intake after recovery from general anesthesia can improve the comfort of children, improve their perioperative metabolic state, reduce thirst and hunger, reduce crying, and thereby help facilitate an earlier recovery.

\section{Limitations}

Children did not drink or eat from $4 \mathrm{~h}$ after returning to the ward, based on standard protocols. There were no statistical differences in thirst or hunger scores from 2 to $4 \mathrm{~h}$ after returning to the ward. Further assessment of oral hydration should be continued after returning to the ward.

\section{Conclusions}

Postoperative oral intake of fluids is very important for children following general anesthesia. This prospective randomized trial provided evidence that administering oral hydration at early post-anesthesia time points was safe in those children, improving satisfaction and decreasing thirst. Perioperative oral intake of fluids is very important for children following general anesthesia.

\section{Abbreviations}

DOH: Late oral hydration; EOH: Early oral hydration; ASA: American Society of Anesthesiology; PACU: Postanesthesia care unit; ChiCTR: Chinese Clinical Trial Registry

\section{Acknowledgments \\ The authors would like to thank Department of Anesthesiology and Department of Pain Management of West China Hospital.}

\section{Authors' contributions}

LY contributed to the conception and design of the study, manuscript writing and final approval of the manuscript. XYR contributed to the design of the study, with emphasis on the statistical analysis and sample size analyses, and final approval of the study. XQZ, TW, BBD, MHW and ANJ contributed to the design of the study, statistics analysis and manuscript writing. All authors have read and approved the final manuscript.

\section{Funding}

Funded by grant 2016ZR0077 from Science \& Technology Department of Sichuan Province (founder: Xiaorong Yin)and grant 2019HXFH069 from 1.3.5 project for disciplines of excellence-Clinical Research Incubation Project, West China Hospital, Sichuan University (founder: Ling Ye. 


\section{Availability of data and materials}

The datasets used and/or analysed during the current study available from the corresponding author on reasonable request.

\section{Ethics approval and consent to participate}

The study was approved by the institutional ethics committee of West China Hospital Sichuan University (Chengdu, China). Written informed consent about the study protocol was obtained from each patient preoperatively.

\section{Consent for publication}

Not applicable.

\section{Competing interests}

The authors declare that they have no competing interests.

\section{Author details}

'Department of Anesthesiology, West China Hospital, Sichuan University/

West China School of Nursing, Sichuan University, Chengdu, Sichuan

Province 610041, People's Republic of China. ${ }^{2}$ Department of Pain

Management, West China Hospital, Sichuan University, Chengdu, Sichuan

Province 610041, People's Republic of China.

Received: 9 December 2019 Accepted: 8 July 2020

Published online: 18 July 2020

\section{References}

1. Ting $L$. Analysis and prevention of concerns in nursing care of patients in anaesthesia recovery room. J Aerospace Med. 2014;25(6):889-90.

2. Xiaoging L. Perianaesthesia management of agitation during general anesthesia recovery period in children. Chin Modern Med. 2010;17(19):94.

3. Yuanfang C. Nursing care of children during anaesthesia recovery period after operation. Chin Foreign Med Res. 2012;10(21):78.

4. Hao L, Li X, Zhao Z. Study on risk factors and prevention and treatment of emergence agitation. Modern Med J China. 2014;16(10):106-8.

5. Mendelson $\mathrm{CL}$. The aspiration of stomach contents into the lungs during obstetric anesthesia. Am J Obstet Gynecol. 1946;52:191-205.

6. Exarhos ND. The importance of $\mathrm{pH}$ and volume in tracheobronchial aspiration. Dis Chest. 1965;47(2):167-9.

7. James CF. Pulmonary aspiration effects of volume and $\mathrm{pH}$ in the rat. Anesth Analg. 1984;63(7):665-8.

8. Xi S. Eye, ear, nose, throat and oral nursing. Beijing: People's Medical Publishing House; 2006. p. 147-50.

9. Weixin C. Surgical nursing. 3rd ed. Beijing: People's Medical Publishing House; 2006. p. 18-46.

10. Xiaoping C. Surgery, vol. 57. Beijing: People's Medical Publishing House; 2006.

11. Chuanzhong D, Ailian W, Zhuo L. Application of early food intake in children after general anesthesia undergoing non-gastrointestinal operation Modern Clin Nurs. 2011;10(6):9.

12. Xiaorong $Y$, Ling $T$, Liao YA. Feasibility of a small mount of water intake at early stage after general anesthesia in children. Chin J Anesthesiol. 2012; 32(3):282-3.

13. Xiaojing $\mathrm{H}$, Nanhai $\mathrm{P}$, Jiwei J. Prolonged fasting before surgery should be abolished in patients undergoing elective surgery. J Clin Med Pract. 2007; 11(5):21-2.

14. Kerz T, Wahlen BM. How long should patients refrain from oral food and fluid intake after general anaesthesia?An assessment of the swallowing reflex of postoperative neurosurgical patients. Minim Invasive Neurosurg. 2004:47(6):378-81.

15. Lu G. Evaluation of the effect of individualized health education on fasting before operation in children. Guangxi Med J. 2008:30(7):1053-4.

\section{Publisher's Note}

Springer Nature remains neutral with regard to jurisdictional claims in published maps and institutional affiliations.

\section{Ready to submit your research? Choose BMC and benefit from:}

- fast, convenient online submission

- thorough peer review by experienced researchers in your field

- rapid publication on acceptance

- support for research data, including large and complex data types

- gold Open Access which fosters wider collaboration and increased citations

- maximum visibility for your research: over $100 \mathrm{M}$ website views per year

At $\mathrm{BMC}$, research is always in progress.

Learn more biomedcentral.com/submissions 